\title{
X-Band Dielectric Resonator Bandpass Filter
}

\author{
M. F. Ain, Z. A. Ahmad, M.A. Othman, I. A. Zubir, \\ S. D. Hutagalung. \\ School of Electrical and Electronics / Material and Mineral \\ Resources Engineering, USM, 14300 Penang, Malaysia. \\ e-mail: mohdfadzilain@yahoo.com.my
}

\author{
A. A. Sulaiman, A. Othman \\ Faculty of Electrical Engineering, \\ UiTM, 40450 Shah Alam, Malaysia \\ e-mail: asari100@yahoo.com
}

\begin{abstract}
This paper presents a new approach of designing a bandpass filter by applying a combination of microstrip and cylindrical shape of dielectric resonators for X-Band application. Three dielectric resonators with a same permittivity and diameter of 60 and $5 \mathrm{~mm}$ respectively are applied in the circuit in order to obtain a wideband of more than $1.0 \mathrm{GHz}$. The interaction between the microstrip transmission line and dielectric resonators increases the coupling effect as well as minimizing the insertion loss in the filter passband. An analysis on the effect of the height of the dielectric resonators has been investigated in order to prove that the new approach contributes more advantages and viable at the desired application band.
\end{abstract}

Keywords- Bandpass filter; Dielectric resonator; wideband.

\section{INTRODUCTION}

Communication systems demand a large number of basestation filters that not only excellent in performance of low loss, but also a good out-of-band spurious performance. A high performance resonator is an important element in many microwave circuits such as filters, amplifiers, couplers, and antennas. There are variety of geometrical resonators have been reported by Virdee [1]. The Dielectric resonator (DR) offers a lot of advantages in increasing the performance of RF and microwave devices which make it an ideal candidate for wireless application; low design profile and wide bandwidth application [2]. However, the performance of the most distributed resonators is limited due to the use of effective dielectric constant and discontinuity of the transmission line. Strip line structures have grasped substantial research interests due to the advantages such as ease of realization both in series or shunt stubs and without require any via holes [3-5]. The design procedure of bandpass filter microstrip lines is well documented in literature [6].

Dielectric resonators are mainly designed to replace resonant cavities in microwave circuits such as filters and oscillators. Dielectric resonator filters are preferable for wireless base stations due to their superior characteristics of a high quality Q-factor and miniaturization. The advantages of dielectric resonators are high temperature stability and ease to be applied. Moreover they can be amenable in multitechnology such as printed circuit and surface mounts technology. Dielectric resonators are also usually shielded to prevent radiation as well as maintain a high-Q that required by filter and oscillator circuits [7].
The most famous filter in microwave applications is the bandpass filter [3]. The filter can be narrow- or wide-bands depend on the applications. A narrow-band bandpass device is designed for stringent specifications of passband insertion loss and stopband rejection. While a wide-band bandpass filter is normally used for high data transmission involving a lot of video and data communications [6]. Their practical realization are varies depend on the applications. For a solution to be practically useful, it needs to be easily manufactured, reliable, add little or no extra cost to the system in a mass production. The solutions also add only a minimum insertion loss to the filter since this attenuation will directly limits the performance of the wireless system.

The DR filters are good for mobile and satellite communications. A typical DR filter consists of a number of dielectric resonators that are mounted in a planar configuration to obtain a good resonant frequency [7]. The relative dielectric constant of the DR in microwave filters generally was chosen from a higher value compared to the base substrate. The primary advantage in using a high dielectric constant is to miniaturize the filter size. The size of DR filter is considerably smaller than the dimension of waveguide filters operate at the same frequency. Furthermore, these DR filters are employed to replace waveguide filters in applications such as satellite communication systems where the planar filters cannot be used due to the inherent of high loss.

In this paper, a novel bandpass filter that consists of three dielectric resonators excited with a microstrip transmission line that used to increase the bandwidth of the design. The idea of using the three dielectric resonators is to generate few additional frequencies which can be merged together to produce a wideband devices, increase the transmitting power and reduce the insertion loss in the passband. The optimum coupling effect in the filter was obtained from the matching position of the resonators on the microstrip line. An analysis on the height of all dielectric resonators also has been done on simulation with the help of CST Microwave Studio while measurement on the S-parameters was done by E8364B Network Analyzer.

\section{DESIGN METHODOLOGY}

The dielectric resonators can increase the Q-factor in a microwave circuit. The size, location and shape of the dielectric resonators including the height and size area will influence the matching of the circuit. In this project, three 